\title{
Skin rash following Administration of Apalutamide in Japanese patients with Advanced Prostate Cancer: an integrated analysis of the phase 3 SPARTAN and TITAN studies and a phase 1 open-label study
}

Hiroji Uemura', Yosuke Koroki ${ }^{2 *}$ (D, Yuki Iwaki ${ }^{3}$, Keiichiro Imanaka ${ }^{4}$, Takeshi Kambara ${ }^{5}$, Angela Lopez-Gitlitz ${ }^{6}$, Andressa Smith ${ }^{6}$ and Hirotsugu Uemura ${ }^{7}$

\begin{abstract}
Background: A higher incidence of apalutamide-related skin rash has been observed in Japanese patients with prostate cancer (PC).
\end{abstract}

Methods: This integrated analysis of data of Japanese patients from 2 global Phase 3 studies, SPARTAN (NCT019462 04; patients with non-metastatic castration-resistant PC [nmCRPC]) and TITAN (NCT02489318; patients with metastatic castration-sensitive PC [mCSPC]), and the Phase 1 study 56021927PCR1008 (NCT02162836; patients with metastatic CRPC [mCRPC]), assessed clinical risk factors of apalutamide-related skin rash as well as the potential correlation with plasma exposure to apalutamide. Kaplan-Meier method was used for time-to-event analyses. Clinical risk factors for skin rash were assessed using odds ratio.

Results: Data from 68 patients (SPARTAN: $n=34$, TITAN: $n=28$, 56021927PCR1008: $n=6$ ) receiving apalutamide $240 \mathrm{mg}$ orally once-daily were analyzed. Rash (13 [19.1\%]) and maculo-papular rash (11 [16.2\%]) were the most frequently reported skin rash. All Grade and Grade 3 skin rash occurred in 35 (51.5\%) and 10 (14.7\%) patients, respectively. Most (85.7\%) skin rash occurred within 4 months of apalutamide initiation and resolved in a median time of 1 month following the use of antihistamines, topical or systemic corticosteroids, with/without apalutamide dose interruptions/reductions. Median time-to-remission of first incidence of rash and maximum grade incidence of rash were 1.0 month (IQR: 0.36-1.81) and 1.0 month (IQR: 0.30-2.43), respectively. No significant clinical risk factors for the incidence of skin rash were observed. Areas under the curve (0-24 h) (AUC $\mathrm{C}_{0-24, \text { ss }}$ ) at steady-state of plasma apalutamide concentration were numerically slightly higher in patients with skin rash than those without.

\footnotetext{
* Correspondence: ykoroki@its.jnj.com

${ }^{2}$ Medical Affairs, Janssen Pharmaceutical K.K., Tokyo, Japan

Full list of author information is available at the end of the article
} 
(Continued from previous page)

Conclusions: No clinical risk factors for rash could be detected. There is a potential correlation between incidence of skin rash and plasma exposure to apalutamide. In general, apalutamide-related skin rash is easily managed, with appropriate treatment with or without dose adjustment.

Trial registration: Retrospective pooled analysis of NCT01946204, NCT02489318, and NCT02162836.

Keywords: Apalutamide, Prostate cancer, Skin rash, Japanese

\section{Background}

Apalutamide, an oral non-steroidal, second-generation, selective inhibitor of the androgen receptor (AR), binds directly to the ligand-binding domain of the AR, thereby preventing AR nuclear translocation and AR-mediated transcription, which in turn induces tumour cell death [1, 2]. Apalutamide is FDA-approved for the treatment of non-metastatic castration-resistant prostate cancer (nmCRPC) and metastatic castration-sensitive prostate cancer (mCSPC), based on data from two pivotal Phase 3 trials, SPARTAN (NCT01946204) and TITAN (NCT024 89318), respectively. In the SPARTAN study, addition of $240 \mathrm{mg}$ apalutamide once-daily (QD) to ongoing androgen-deprivation therapy (ADT) showed a significant increase in metastasis-free survival (MFS) (> 2 years) when compared to placebo, with an overall maintenance of health-related quality of life. However, the incidence of skin rash was higher in the apalutamide group compared to placebo (23.8\% vs $5.5 \%)$ [3]. Similarly, in the TITAN study, skin rash was more frequently observed in the apalutamide group compared to placebo (27.1\% vs $8.5 \%)$, although significant improvements in the dual primary endpoints of overall survival (OS) and radiographic progression-free survival (rPFS) were reported [4]. Rash is a grouped term that includes macular rash, maculopapular rash, butterfly rash, erythematous rash, generalized rash, papules, papular rash, pruritic rash, pustular rash, systemic lupus erythematosus rash, erythema multiforme, stomatitis, and urticaria among others. It is important to note that the most commonly observed apalutamide-related rash is not graded based on severity but on body surface area (BSA) involved. Therefore, to be defined as a Grade 3 rash, it must cover $>30 \%$ BSA, regardless of concomitant symptoms, if any.

Interestingly, the subgroup analysis of Japanese patients in both the SPARTAN and TITAN studies showed that a greater proportion of patients treated with apalutamide developed skin rash compared with placebo (SPARTAN subgroup: $56.0 \%$ vs $0.0 \%$; TITAN subgroup: $50.0 \%$ vs $8.7 \%$ ) [5], [Uemura et al., International Journal of Urology, submitted], with the incidence in Japanese patients being nearly double the incidence observed in the global populations of the two studies. In a Phase 1 trial (56021927PCR1008, referred hereafter as PCR1008) conducted to study the safety, efficacy and pharmacokinetic (PK) profile of apalutamide in Japanese patients with metastatic CRPC (mCRPC) (NCT02162836) [6], skin rash was observed in $2 / 6$ (33.3\%) patients. Taken together, these data suggest that apalutamide-related skin rash is more frequently observed in Japanese patients. However, the relationship between patient characteristics and apalutamide-related skin rash is not clear. Moreover, the clinical risk factors for apalutamide-related skin rash and the relationship between apalutamide exposure and skin rash remains to be elucidated.

Therefore, we conducted an integrated analysis of data from Japanese patients in the SPARTAN and TITAN studies, along with data from the PCR1008 study primarily to investigate the incidence rate, types, severity and management of skin rash in Japanese subpopulation. Data from patients who developed skin rash were further analyzed to assess if the grade of skin rash had a correlation with the extent of exposure to apalutamide. In addition, various clinical risk factors, such as Gleason score and previous therapy were evaluated vis-à-vis their contribution to the incidence of skin rash.

\section{Methods}

A pooled analysis of data from Japanese patients who were administered apalutamide in two global Phase 3 studies, SPARTAN and TITAN, and a Phase 1 study (PCR1008) was conducted. The study protocols and informed consent forms for the original trials had been reviewed and approved by their respective Independent Ethics Committee or Institutional Review Board. Studies were conducted in accordance with ethical principles outlined in the Declaration of Helsinki and were consistent with International Conference of Harmonization, Good Clinical Practices guidelines, and applicable regulatory requirements. Patients or their legally acceptable representatives provided written informed consent before enrollment. The original trials (SPARTAN, TITAN and PCR1008) were conducted and funded by Janssen Research \& Development, LLC and Janssen Pharmaceuticals K.K.

The study design for each of the 3 studies is briefly presented below: 


\section{SPARTAN}

A total of 1207 patients with $n m C R P C$, receiving ongoing ADT, were randomly assigned (2:1) to either apalutamide (240 mg, QD, orally) or matched placebo, and treated until disease progression, withdrawal of consent, unacceptable toxicity or death. The primary efficacy endpoint was MFS. Secondary efficacy endpoints were time to metastasis, PFS, time to symptomatic progression, time to initiation of cytotoxic chemotherapy and OS.

\section{TITAN}

A total of 1052 patients with $\mathrm{mCSPC}$ were randomized (1:1) to receive apalutamide (240 mg, QD, orally) plus ADT or placebo plus ADT, in this double-blind, Phase 3 trial. The dual primary endpoints were rPFS and OS. Secondary efficacy endpoints were time to cytotoxic chemotherapy, pain progression, chronic opioid use, and skeletal-related event.

\section{Population pharmacokinetic analysis in SPARTAN and TITAN} A population $\mathrm{PK}$ analyses were performed using all available drug concentration data from SPARTAN and TITAN studies. The population PK model for plasma concentrations of apalutamide and its active metabolite, N-desmethyl apalutamide, was developed using nonlinear mixed-effects modeling. Individual exposure metrics i.e., areas under the curve after $24 \mathrm{~h}$ at steadystate $\left(\mathrm{AUC}_{0-24}\right.$, ss $\left.\left[\mu \mathrm{g}^{*} \mathrm{~h} / \mathrm{mL}\right]\right)$ of plasma concentration, for apalutamide and $\mathrm{N}$-desmethyl apalutamide were derived using post-hoc estimates of the PK parameters. $\mathrm{AUC}_{0-24 \text {, ss }}$ was calculated based on an average dose up to time of the first rash event considering possible dose modifications. The details of the population PK model was reported in a separate article [7].

\section{PCR1008}

A total of 6 patients were included in this Phase 1, open-label, multi-center study to analyze the safety, tolerability, and PK profile of apalutamide (240 mg, QD, orally) in Japanese patients with $\mathrm{MCRPC}$ who were on ADT background therapy (either medical or surgical castration). Patients received a single apalutamide $240 \mathrm{mg}$ dose on Day 1 of the PK week. Patients were reassessed for safety. If no safety signals were reported, patients received apalutamide $240 \mathrm{mg}$ QD until disease progression, unacceptable toxicity, withdrawal of consent, or death, whichever occurred first. Safety was assessed. All adverse events (AEs) were graded according to National Cancer Institute Common Terminology Criteria for Adverse Events (NCI CTCAE), v4.0.3 in the SPARTAN and TITA $\mathrm{N}$ studies, while NCI CTCAE v4.0 was used in PCR1008.

\section{Analysis}

Incidence rate, type of rash, severity, management The incidence rate, type and grade of rash, along with the treatments for the management of rash in each of the 3 studies was analyzed.

Time-to-event analysis The time-to-event analysis for rash was performed using the Kaplan-Meier method. Kaplan-Meier curves were generated for time-to-rash (All Grade and Grade 3 or more) and time-to-remission (first incidence, All Grade; first incidence, Grade 3 or more; last incidence, All Grade; and last incidence, Grade 3 or more) using individual patient-level data.

Clinical risk factors Odds ratio and 95\% confidence interval was used to assess whether factors including age; height; weight; body mass index; Eastern Cooperative Oncology Group Performance Status (ECOG PS); time from initial diagnosis to first dose, Gleason score at initial diagnosis; baseline prostate-specific antigen (PSA), alkaline phosphatase (ALP), hemoglobin, or lactate dehydrogenase levels; and previous local treatment, firstgeneration antiandrogens, or chemotherapy were related to the incidence of rash.

Pooled data for baseline characteristics, treatment duration, dose reduction, dose interruption, dose discontinuation, incidence of rash and management of rash are presented using descriptive statistics.

Plasma exposure and incidence of rash The relationships between the occurrence (by grade) of skin rash and the plasma exposure $\left(\mathrm{AUC}_{0-24, \mathrm{ss}}\right)$ to apalutamide or $\mathrm{N}$-desmethyl apalutamide were explored using boxplots. All exposure analyses were done based on data from the SPARTAN and TITAN studies.

\section{Results}

In total, 1207 patients in SPARTAN and 1052 patients in TITAN were enrolled globally, of which 55 and 51 patients were Japanese, respectively. This integrated analysis included data from total 68 Japanese patients (SPARTAN: $n=34$, TITAN: $n=28$, PCR1008: $n=6$ ) who had received apalutamide $240 \mathrm{mg}$ QD orally (Fig. 1).

\section{Baseline characteristics}

Overall, the median age of patients was 77 years. Since the disease status at baseline was different in SPARTAN, TITAN, and PCR1008 studies, some of the baseline characteristics were not comparable between the three groups, with the median time from initial diagnosis, PSA levels, treatment durations, and ALP levels varying between the 3 patient populations. Median time from 


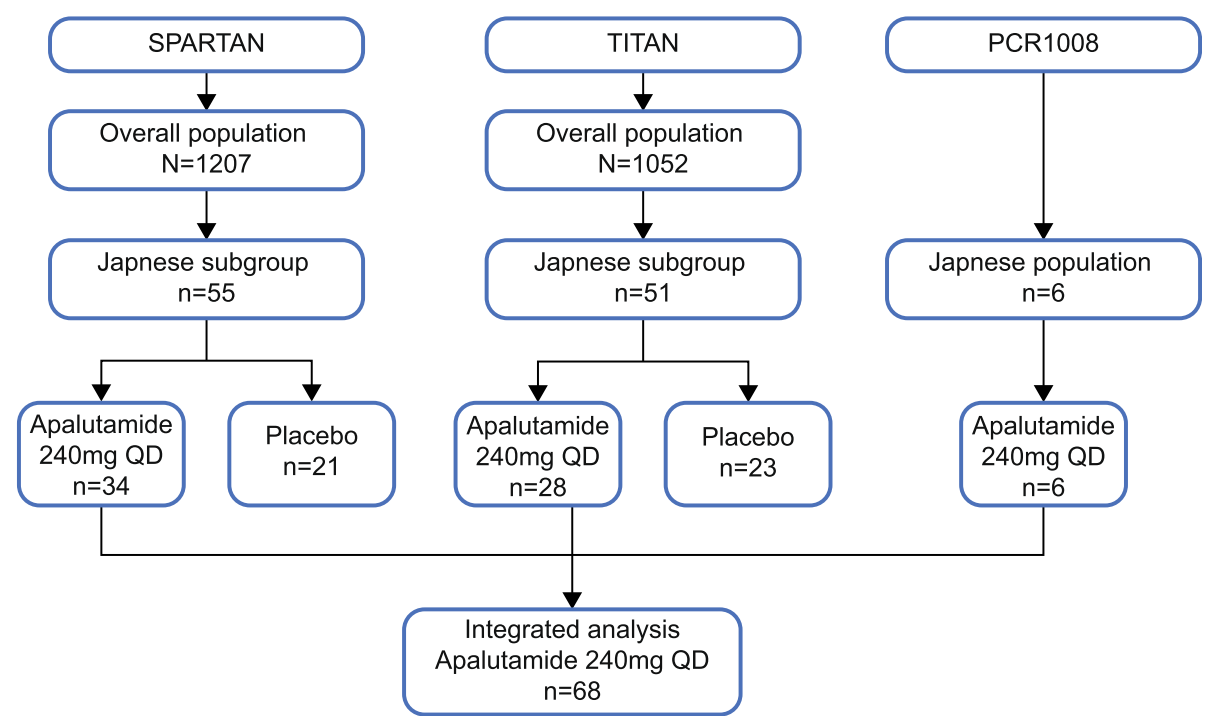

Fig. 1 Flow diagram representing patient recruitment from SPARTAN, TITAN and PCR1008 studies

initial diagnosis to first dose was shortest in TITAN (2.19 months), followed by PCR1008 (68.89 months) and SPARTAN (87.10 months); with median PSA level $[\mathrm{ng} / \mathrm{mL}]$ at baseline of $11.51,54.42$ and 4.35 , respectively. All patients had received prior hormonal therapy (Table 1).

\section{Incidence rate, types of rash, severity}

In the global SPARTAN and TITAN studies, the overall incidence of skin rash in the apalutamide group was 191/ $803(23.8 \%)$ and $142 / 524$ (27.1\%), respectively, with the combined incidence rates of the most commonly reported rash in the 2 studies, i.e., rash, generalized rash, and maculo-papular rash, being 167/1327 (12.6\%), 53/1327 (4.0\%), and 60/1327 (4.5\%), respectively (Supplementary Table 1). In the present integrated analysis of Japanese patients from SPARTAN and TITAN, and PCR1008, skin rash was observed in 35/68 (51.5\%) of the patients, and the incidence rates of rash (13/68 [19.1\%]), generalized rash $(11 / 68$ [16.2\%]), and maculo-papular rash (11/68 [16.2\%]) (Table 2) were also higher than that observed in the global studies. Also, the incidence rate of the less commonly observed rash, erythema multiforme and stomatitis, were higher in Japanese patients (3/68 [4.4\%], each) compared to their combined incidence rates in the global SPARTAN and TITAN studies (6/1327 [0.45\%] and 10/1327 [0.75\%], respectively) (Supplementary Table 1 ).

In the global SPARTAN and TITAN studies, the incidence of Grade 1 skin rash was 69/803 (8.6\%) and $57 / 524$ (10.9\%), respectively, in the apalutamide group, while the corresponding values for Grade 2 events were $80 / 803 \quad(10.0 \%)$ and 52/524 (9.9\%), respectively
(Supplementary Table 1). In the Japanese patients analyzed for this study, the incidence of Grade 1 rash was similar between the groups (SPARTAN: 4/34 [11.8\%], TITAN: 4/28 [14.3\%], PCR1008: 1/6 [16.7\%]), with overall incidence being 9/68 (13.2\%); Grade 2 rash were more frequently observed in the SPARTAN study (10/34 [29.4\%]) compared to the TITAN (5/28 [17.9\%]) and PCR1008 (1/6 [16.7\%]) studies; while Grade 3 rash was observed only in the SPARTAN (5/34 [14.7\%]) and TITAN (5/28 [17.9\%]) studies. Overall, Grade 3 skin rash occurred in $10 / 68(14.7 \%)$ patients in the current integrated analysis, compared to $42 / 803(5.2 \%)$ and 33/524 (6.3\%) patients in the SPARTAN and TITAN global studies, respectively. (Supplementary Table 1) There were no Grade 4 or 5 rashes due to the grading criteria used for reporting the types of rash observed.

\section{Management of Rash}

All Japanese patients with skin rash (35/68 [51.1\%]) received supportive medications; topical corticosteroid was the most common (27/35 [77.1\%]), followed by oral antihistamine and systemic corticosteroid (18/35 [51.4\%] and $3 / 35$ [8.6\%], respectively) (Table 3 ). In comparison, in the global SPARTAN and TITAN studies, antihistamines were required in $67 / 191$ (35.1\%) and 54/142 (38.0\%) patients, systemic corticosteroids in 33/191 (17.3\%) and 29/ $142(20.4 \%)$ patients, while topical corticosteroids were administered in 65/191 (34.0) and 61/142 (43.0\%) patients, respectively (Supplementary Table 2).

Drug interruptions and dose reductions were required in $18 / 35(51.4 \%)$ and $7 / 35(20.0 \%)$ patients, respectively, with treatment discontinuation required in $5 / 35$ (14.3\%) 
Table 1 Baseline Characteristics (Safety Set)

\begin{tabular}{|c|c|c|c|c|}
\hline Categories & SPARTAN & TITAN & PCR1008 & Total \\
\hline Number of patients in safety analysis set, $n$ & 34 & 28 & 6 & 68 \\
\hline Median age, years (range) & $79.00(61-90)$ & $73.00(47-89)$ & $78.00(70-85)$ & $77.00(47-90)$ \\
\hline Median weight, kg (range) & $61.90(45.5-84.0)$ & $63.55(40.6-89.2)$ & $57.30(49.8-62.6)$ & $61.80(40.6-89.2)$ \\
\hline Median height, cm (range) ${ }^{a}$ & $163.50(140.0-176.4)$ & $165.20(151.7-172.8)$ & $163.55(147.0-165.9)$ & $164.00(140.0-176.4)$ \\
\hline \multicolumn{5}{|l|}{ ECOG PS ${ }^{a}, n(\%)$} \\
\hline 0 & $30(88.24)$ & $25(89.29)$ & $5(83.33)$ & $60(88.24)$ \\
\hline 1 & $4(11.76)$ & $3(10.71)$ & $1(16.67)$ & $8(11.76)$ \\
\hline $\begin{array}{l}\text { Median time from initial diagnosis to first dose, } \\
\text { month (range) }\end{array}$ & $87.10(11.01-176.10)$ & $2.19(1.28-74.15)$ & $68.89(9.23-132.72)$ & $46.42(1.28-176.10)$ \\
\hline \multicolumn{5}{|l|}{ GS at initial diagnosis, $\mathrm{n}(\%)$} \\
\hline$\leq 7$ & $9(26.47)$ & $1(3.57)$ & $2(33.33)$ & $12(17.65)$ \\
\hline$\geq 8$ & $25(73.53)$ & $27(96.43)$ & $4(66.67)$ & $56(82.35)$ \\
\hline Median PSA at baseline, $\mathrm{ng} / \mathrm{mL}$ (range) & $4.35(0.45-21.71)$ & $11.51(0.04-457.64)$ & $54.42(8.92-310.11)$ & $5.73(0.04-457.64)$ \\
\hline Median Hemoglobin at baseline, $\mathrm{ng} / \mathrm{mL}$ (range) & $12.95(9.4-15.5)$ & $13.45(10.2-15.9)$ & $11.95(9.9-15.0)$ & $13.10(9.4-15.9)$ \\
\hline Median LDH at baseline, U/L (range) & - & $198.00(123-272)$ & $192.00(115-456)$ & $197.50(115-456)$ \\
\hline Median ALP at baseline, U/L (range) & $73.50(33-137)$ & $104.50(56-1149)$ & $204.50(102-331)$ & $87.00(33-1149)$ \\
\hline \multicolumn{5}{|l|}{ Disease status, $\mathbf{n}$} \\
\hline $\mathrm{nmCRPC}$ & 55 & - & - & 55 \\
\hline mCSPC & - & 51 & - & 51 \\
\hline mCRPC & - & - & 6 & 6 \\
\hline \multicolumn{5}{|l|}{ Tumor volume $^{\mathrm{b}}, \mathrm{n}(\%)$} \\
\hline High & - & $18(64.29)$ & - & $18(64.29)$ \\
\hline Low & - & $10(35.71)$ & - & $10(35.71)$ \\
\hline \multicolumn{5}{|l|}{ Previous treatment, $\mathrm{n}(\%)$} \\
\hline Local treatment & $21(61.76)$ & $1(3.57)$ & $2(33.33)$ & $24(35.29)$ \\
\hline $\mathrm{RP}$ & $5(14.71)$ & $1(3.57)$ & $1(16.67)$ & $7(10.29)$ \\
\hline RT & $19(55.88)$ & $1(3.57)$ & $1(16.67)$ & $21(30.88)$ \\
\hline Hormonal treatment & $34(100.00)$ & $28(100.00)$ & $6(100.00)$ & $68(100.00)$ \\
\hline LHRHa & $34(100.00)$ & $28(100.00)$ & $5(83.33)$ & $67(98.53)$ \\
\hline Orchiectomy & $1(2.94)$ & $2(7.14)$ & $1(16.67)$ & $4(5.88)$ \\
\hline 1st generation AA & $33(97.06)$ & $15(53.57)$ & $6(100.00)$ & $54(79.41)$ \\
\hline Other & $2(5.88)$ & $0(0.00)$ & $0(0.00)$ & $2(2.94)$ \\
\hline Chemotherapy & $0(0.00)$ & $0(0.00)$ & $0(0.00)$ & $0(0.00)$ \\
\hline Other & $3(8.82)$ & $0(0.00)$ & $2(33.33)$ & $5(7.35)$ \\
\hline \multicolumn{5}{|l|}{ Average dose of Apalutamide } \\
\hline Mean dose, mg (SD) & $223.94(28.91)$ & $214.07(44.90)$ & $225.84(25.76)$ & $220.04(36.05)$ \\
\hline Median dose, mg (range) & $236.55(108.6-240.0)$ & $239.02(90.6-240.0)$ & $236.10(174.2-240.0)$ & $238.27(90.6-240.0)$ \\
\hline
\end{tabular}

AA Antiandrogen, ALP Alkaline phosphatase, ECOG Eastern Cooperative Oncology Group, GS Gleason score, LDH Lactate dehydrogenase LHRHa, hormone-releasing hormone antagonist, $n m C R P C$ Non-metastatic castration resistant prostate cancer, $m C S P C$ Metastatic castration sensitive prostate cancer, $m C R P C$ Metastatic castration resistant prostate cancer, PSA Prostate-specific antigen, RP Radical prostatectomy, RT Radiotherapy, SD Standard deviation

${ }^{a}$ : count PCR1008 at screening

b. Denominator of proportion is TITAN's safety analysis set

patients. Among patients receiving apalutamide who developed skin rash in the global SPARTAN study, dose interruptions, does reductions, and treatment discontinuations were reported in 55/191 (28.8\%), 22/191 (11.5\%), and 19/191 (9.9\%), patients, respectively, while the corresponding values in the global population from the TITAN study were $44 / 142$ (31.0\%), 28/142 (19.7\%), and 12/142 (8.5\%) (Supplementary Table 2). 
Table 2 Types of Skin Rash in Apalutamide-treated Patients

\begin{tabular}{lllll}
\hline Analysis Set (N=68) & $\begin{array}{l}\text { Total } \\
\mathrm{n}(\%)\end{array}$ & $\begin{array}{l}\text { Grade 1 } \\
\mathrm{n}(\%)\end{array}$ & $\begin{array}{l}\text { Grade 2 } \\
\mathrm{n}(\%)\end{array}$ & $\begin{array}{l}\text { Grade 3 } \\
\mathrm{n}(\%)\end{array}$ \\
\hline Rash & $35(51.47)$ & $9(13.23)$ & $16(23.52)$ & $10(14.70)$ \\
Rash & $13(19.11)$ & $8(11.76)$ & $4(5.88)$ & $1(1.47)$ \\
Rash maculo-papular & $11(16.17)$ & $2(2.94)$ & $6(8.2)$ & $3(4.41)$ \\
Rash generalised & $11(16.17)$ & $1(1.47)$ & $7(10.29)$ & $3(4.41)$ \\
Erythema multiforme & $3(4.41)$ & 0 & $1(1.47)$ & $2(2.94)$ \\
Stomatitis & $3(4.41)$ & $1(1.47)$ & $2(2.94)$ & 0 \\
Urticaria & $2(2.94)$ & $2(2.94)$ & 0 & 0 \\
Blister & $1(1.47)$ & $1(1.47)$ & 0 & 0 \\
Drug eruption & $1(1.47)$ & 0 & 0 & $1(1.47)$ \\
Rash macular & $1(1.47)$ & 0 & 0 & $1(1.47)$ \\
Skin erosion & $1(1.47)$ & $1(1.47)$ & 0 & 0 \\
Skin exfoliation & $1(1.47)$ & $1(1.47)$ & 0 & 0 \\
\hline
\end{tabular}

\section{Time-to-event analyses}

In the integrated analysis, the median time to onset of first incidence of rash of any grade in Japanese patients was 66 days, with time to incidence of first Grade 3 rash being 45 days and 9/10 (90.0\%) Grade 3 events being reported in the first 4 months. The median time for first incidence of Grade 3 rash was 52 days and 38 days in the Japanese patients from SPARTAN and TITAN, respectively. In comparison, in the global population from the SPARTAN and TITAN studies, the median time to first incidence of skin rash were 82 days and 80.5 days, respectively (Supplementary Table 2). The median time for remission of first incidence of any grade in Japanese patients was 1.0 month (Interquartile range [IQR]: 0.36-1.81) (Fig. 2). In the global population of the SPARTAN study, skin rash of any grade resolved for $81 \%$ of the patients within 59.5 days, while the median time to resolution of skin rash of any grade in the TITAN study was 100 days (Supplementary Table 2). The time-toremission of Grade 3 rash was 35 days and 17 days in the
Japanese patients from SPARTAN and TITAN, respectively, with 1.0 month (IQR: $0.30-2.43)$ required for remission in the integrated analysis (Fig. 3). Some patients experienced skin rash more than once during apalutamide treatment. Even if patients resolved first incidence of rash, there was the potential for worsening in the second incidence of rash. Therefore, we assessed these two types of KM curves. KM figures for time-to-rash (All grade) (Supplementary Fig. 1), time-to-rash ( $\geq$ Grade3) (Supplementary Fig. 2), time-to-remission of first incidence ( $\geq$ Grade3) (Supplementary Fig. 3), and time-toremission of maximum grade ( $\geq$ Grade3) (Supplementary Fig. 4) are included in the supplementary files.

\section{Clinical risk factors}

A number of clinical risk factors that could potentially affect the incidence of rash were assessed, including Eastern Cooperative Oncology Group Performance Status (ECOG PS), time from initial diagnosis to first dose, Gleason score, and previous treatments (Table 4). However, none of the factors were found to be significantly linked to the incidence of skin rash.

\section{Pharmacokinetic analysis}

$\mathrm{AUC}_{0-24}$, ss of apalutamide were numerically slightly higher in patients with skin rash than those without; however, this did not significantly impact the grade of skin rash (Fig. 4a). No correlation was apparent with $\mathrm{AUC}_{0-24, \text { ss }}$ of $\mathrm{N}$-desmethyl apalutamide (Fig. 4b).

\section{Discussion}

Apalutamide-related skin rash has been observed in previous studies, with a higher incidence of skin rash observed in the Japanese subpopulation compared to overall global population. In the global SPARTAN and TITAN studies, and the Japanese subpopulation analysis from these studies [5], [Uemura et al., International Journal of Urology, submitted], Grade 3 rash (covering $>30 \%$ of the body

Table 3 Rash Management

\begin{tabular}{|c|c|c|c|c|}
\hline Analysis Set $(\mathrm{N}=68)$ & SPARTAN & TITAN & PCR1008 & Total \\
\hline Number of patients in safety analysis set, $n$ & 34 & 28 & 6 & 68 \\
\hline Rash, n (\%) & $19(55.88)$ & $14(50.00)$ & $2(33.33)$ & $35(51.47)$ \\
\hline \multicolumn{5}{|c|}{ Patients who received supportive care for rash, $n(\%)$} \\
\hline Oral antihistamine & $11(57.89)$ & $6(42.86)$ & $1(50.00)$ & $18(51.43)$ \\
\hline Systemic corticosteroid & $0(0.00)$ & $3(21.43)$ & $0(0.00)$ & $3(8.57)$ \\
\hline Topical corticosteroid & $13(68.42)$ & $13(92.86)$ & $1(50.00)$ & $27(77.14)$ \\
\hline Drug interruption & $11(57.89)$ & $6(42.86)$ & $1(50.00)$ & $18(51.43)$ \\
\hline Dose reduction & $4(21.05)$ & $3(21.43)$ & $0(0.00)$ & $7(20.00)$ \\
\hline Drug discontinuation & $3(15.79)$ & $2(14.29)$ & $0(0.00)$ & $5(14.29)$ \\
\hline Other & $2(10.53)$ & $1(7.14)$ & $0(0.00)$ & $3(8.57)$ \\
\hline
\end{tabular}




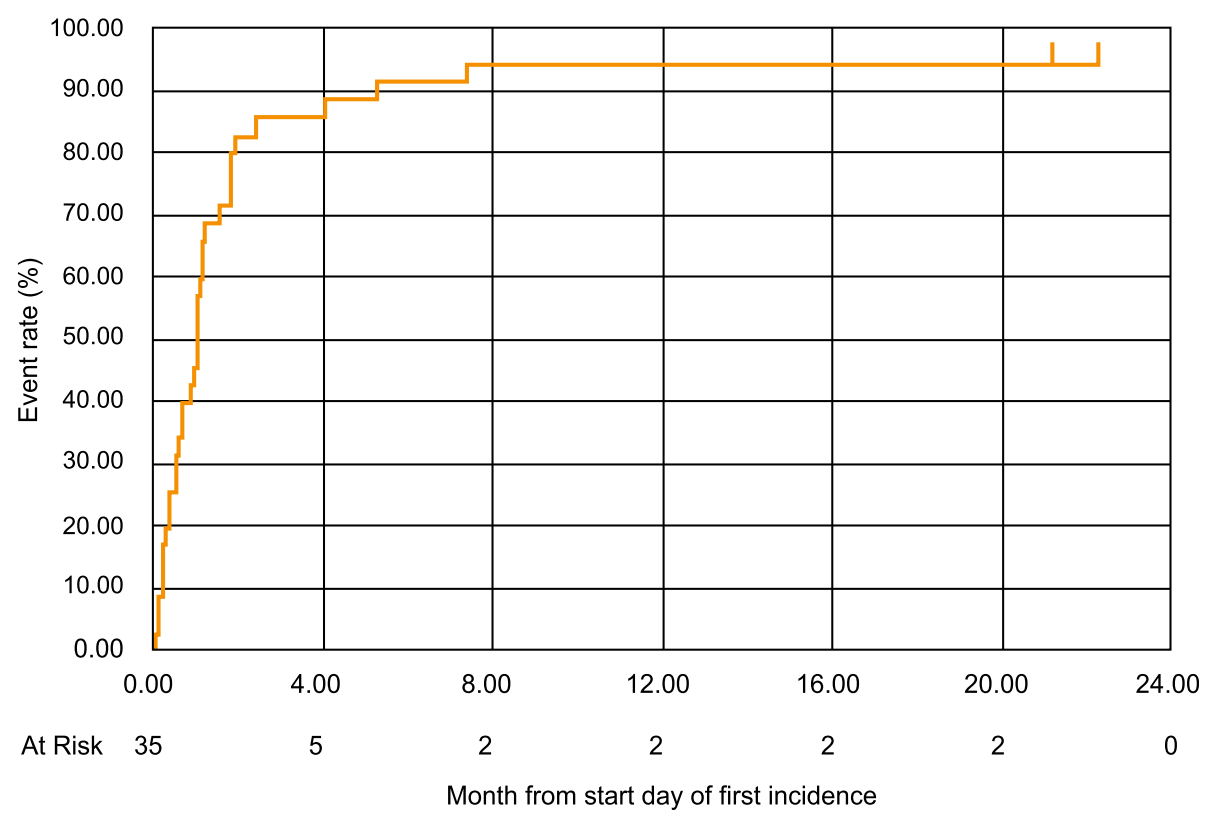

Fig. 2 Kaplan-Meier plot for Time-to-Remission of First Incidence of rash (All Grade). Event: remission of first incidence of rash. Censor: not remission of first incidence of rash at the end of follow-up

surface) was observed more frequently with apalutamide [3, 4]. In the current integrated analysis, the incidence of Grade 3 rash (14.7\%) was also higher than in the global SPAR TAN and TITAN studies (combined incidence, 5.7\%). This suggests that both the frequency and grade of skin rash, across different disease status, are higher in Japanese patients compared to the global population with apalutamide treatment. Consequently, treatment discontinuation due to rash, which is recommended if oral corticosteroids are required for $>28$ days, was required in $14.3 \%$ patients in the current integrated analysis compared with $2.3 \%$ patients in the global population [3, 4]. Most Grade 1/2 rash resolved following the use of oral antihistamines, topical or systemic corticosteroids, with or without apalutamide dose

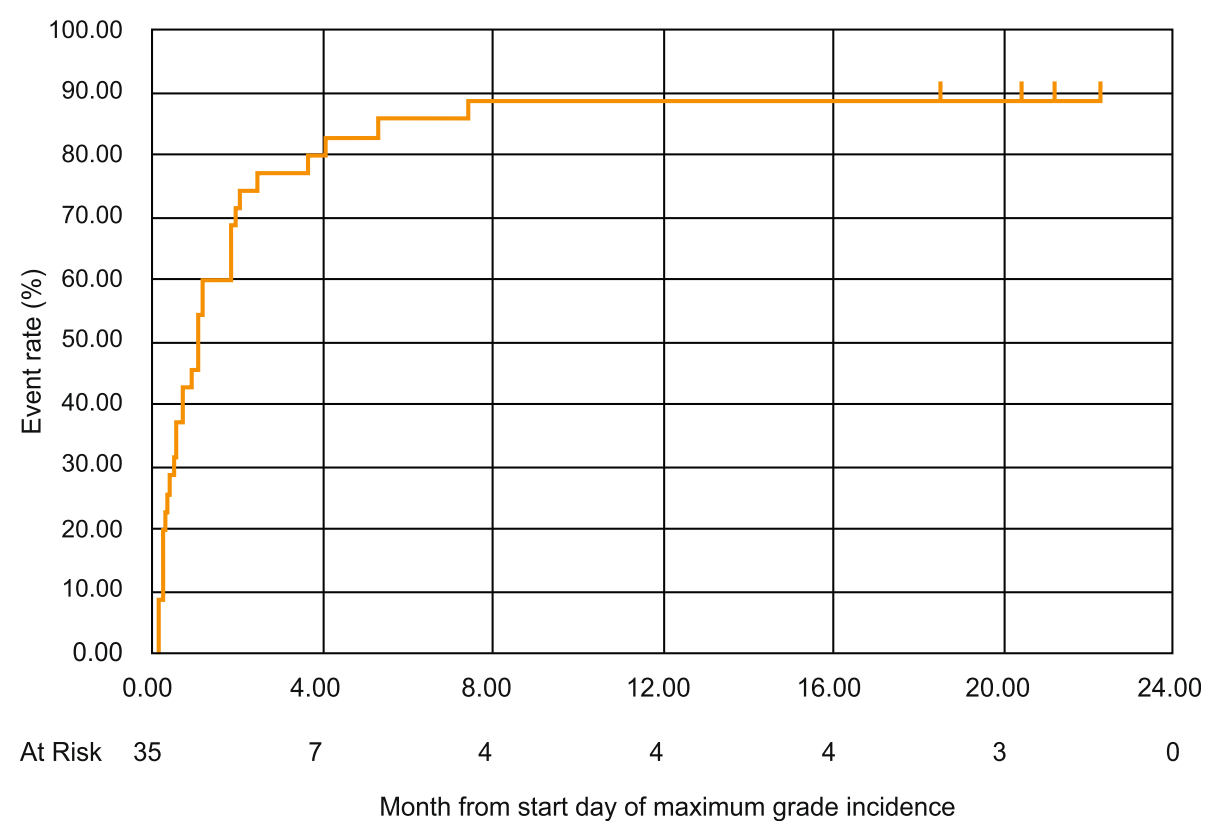

Fig. 3 Kaplan-Meier plot for Time-to-Remission of Maximum Grade Incidence of rash (All grade). Event: remission of maximum Grade incidence of rash. Censor: not remission of maximum Grade incidence of rash at the end of follow-up 
Table 4 Odds Ratio (All Grade), Target Population: Safety

\begin{tabular}{|c|c|c|c|c|c|c|c|}
\hline \multirow[t]{2}{*}{ Categories } & & \multirow{2}{*}{$\begin{array}{l}\text { Number } \\
\text { of } \\
\text { Patients }\end{array}$} & \multicolumn{2}{|l|}{ Rash, n (\%) } & \multirow[t]{2}{*}{$95 \% \mathrm{Cl}$} & \multirow{2}{*}{$\begin{array}{l}\text { Odds } \\
\text { Ratio }\end{array}$} & \multirow{2}{*}{$\begin{array}{l}p^{-} \\
\text {value }\end{array}$} \\
\hline & & & Yes & No & & & \\
\hline \multicolumn{2}{|l|}{ Number of patients in safety analysis set, $n$} & 68 & $35(51.47)$ & $33(48.5)$ & & & \\
\hline \multirow[t]{2}{*}{ Age class 1 , year } & $<65$ & 5 & $3(60.00)$ & $2(40.00)$ & & & \\
\hline & $\geq 65$ & 63 & $32(50.79)$ & $31(49.20)$ & $0.11-4.40$ & 0.69 & 0.693 \\
\hline \multirow[t]{2}{*}{ Age class 2, year } & $<75$ & 28 & $13(46.43)$ & $15(53.57)$ & & & \\
\hline & $\geq 75$ & 40 & $22(55.00)$ & $18(45.00)$ & $0.53-3.72$ & 1.41 & 0.487 \\
\hline \multirow[t]{2}{*}{ Baseline weight } & $<$ Median & 34 & $16(47.06)$ & $18(52.94)$ & & & \\
\hline & $\geq$ Median & 34 & 19 (55.88) & $15(44.12)$ & $0.55-3.70$ & 1.43 & 0.467 \\
\hline \multirow[t]{2}{*}{ Baseline height } & $<$ Median & 34 & $15(44.12)$ & 19 (55.88) & & & \\
\hline & $\geq$ Median & 34 & $20(58.82)$ & $14(41.18)$ & $0.69-4.73$ & 1.81 & 0.227 \\
\hline \multirow[t]{2}{*}{ Body mass index $\left(\mathrm{kg} / \mathrm{m}^{2}\right)$} & $<25$ & 48 & $22(45.83)$ & $26(54.17)$ & & & \\
\hline & $\geq 25$ & 20 & $13(65.00)$ & $7(35.00)$ & $0.75-6.46$ & 2.19 & 0.154 \\
\hline \multirow[t]{2}{*}{ ECOG PS } & 0 & 60 & $30(50.00)$ & $30(50.00)$ & & & \\
\hline & 1 & 8 & $5(62.50)$ & $3(37.50)$ & $0.37-7.61$ & 1.67 & 0.510 \\
\hline \multirow[t]{2}{*}{ Time from initial diagnosis to first dose } & $<$ Median & 34 & $14(41.18)$ & $20(58.82)$ & & & \\
\hline & $\geq$ Median & 34 & $21(61.76)$ & $13(38.24)$ & $0.87-6.10$ & 2.31 & 0.092 \\
\hline \multirow[t]{3}{*}{ GS at initial diagnosis } & $\leq 7$ & 12 & $9(75.00)$ & $3(25.00)$ & & & \\
\hline & $\geq 8$ & 56 & $26(46.43)$ & $30(53.57)$ & $0.07-1.18$ & 0.29 & 0.084 \\
\hline & Unknown & 0 & 0 & 0 & - & - & \\
\hline \multirow[t]{2}{*}{ Baseline PSA } & $<$ Median & 34 & $21(61.76)$ & $13(38.24)$ & & & \\
\hline & $\geq$ Median & 34 & $14(41.18)$ & $20(58.82)$ & $0.16-1.15$ & 0.43 & 0.092 \\
\hline \multirow[t]{2}{*}{ Baseline Hemoglobin } & High & 68 & $35(51.47)$ & $33(48.53)$ & & & \\
\hline & Normal or Low & 0 & 0 & 0 & - & - & - \\
\hline \multirow[t]{2}{*}{ Baseline LDH } & High & 7 & $2(28.57)$ & $5(71.43)$ & & & 0.282 \\
\hline & Normal or Low & 27 & $14(51.85)$ & $13(48.15)$ & $0.44-16.37$ & 2.69 & \\
\hline \multirow[t]{2}{*}{ Baseline ALP } & High & 13 & $4(30.77)$ & $9(69.23)$ & & & \\
\hline & Normal or Low & 55 & $31(56.36)$ & $24(43.64)$ & $0.80-10.59$ & 2.91 & 0.106 \\
\hline \multirow[t]{2}{*}{ Previous local treatment } & No & 44 & $21(47.73)$ & $23(52.27)$ & & & \\
\hline & Yes & 24 & $14(58.33)$ & $10(41.67)$ & $0.56-4.19$ & 1.53 & 0.404 \\
\hline \multirow[t]{2}{*}{ Previous 1st generation AA } & No & 14 & $9(64.29)$ & $5(35.71)$ & & & \\
\hline & Yes & 54 & $26(48.15)$ & $28(51.85)$ & $0.15-1.74$ & 0.52 & 0.286 \\
\hline \multirow[t]{2}{*}{ Previous chemotherapy } & No & 68 & $35(51.47)$ & $33(48.53)$ & & & \\
\hline & Yes & 0 & 0 & 0 & - & - & - \\
\hline
\end{tabular}

AA Antiandrogen, ALP Alkaline phosphatase, ECOG PS Eastern Cooperative Oncology Group Performance Status, GS Gleason score, LDH Lactate dehydrogenase, PSA Prostate-specific antigen, Cl Confidence interval

interruption or reduction, following which, patients were able to continue apalutamide treatment. Based on the current analysis, it is difficult to conclude whether supportive treatment or dose interruption was more effective in resolving the different grades of skin rash.

In our study, the median time to first incidence of rash was 66 days, and it resolved in a median time of 1.0 month. When compared to SPARTAN, both the onset and resolution of rash were faster in Japanese patients. These data require further investigation since the overall incidence of skin rashes was higher in the Japanese patients, yet their time to incidence and resolution was faster. Interestingly, in our study, the median time to first remission was 1.0 month, but the median time-toremission of last incidence was 3.6 months, suggesting that some patients experienced several incidents of skin rash. However, the median time-to-remission of the worst grade (Grade 3) of skin rash was 1.0 month. Therefore, even if some patients experienced several incidents of skin rash, the worst grade of skin rash remitted within 1.0 


\section{a) Apalutamide}

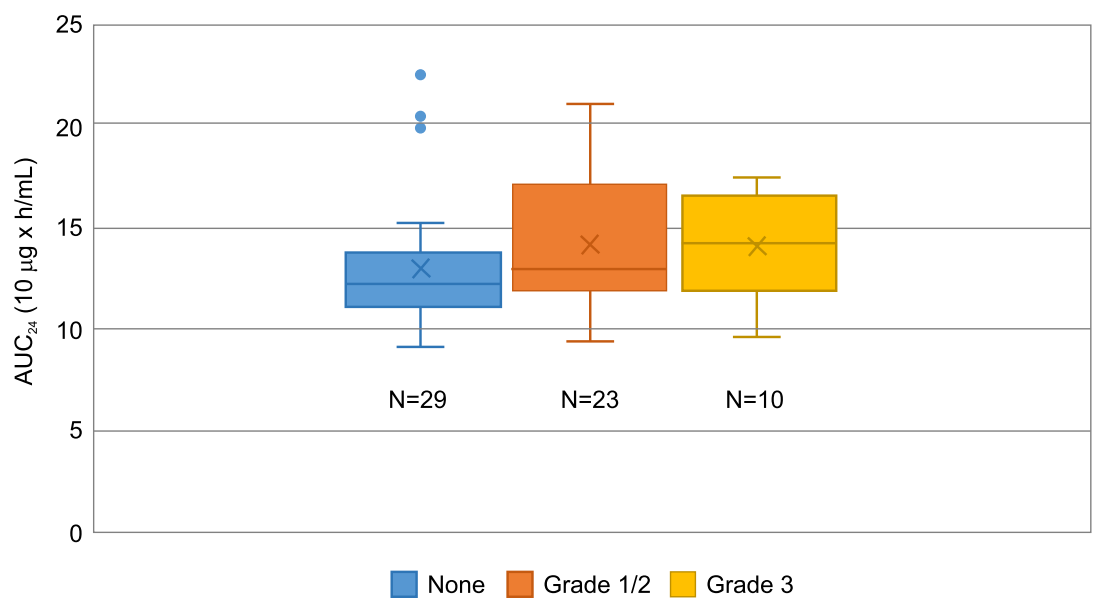

b) N-desmethyl apalutamide

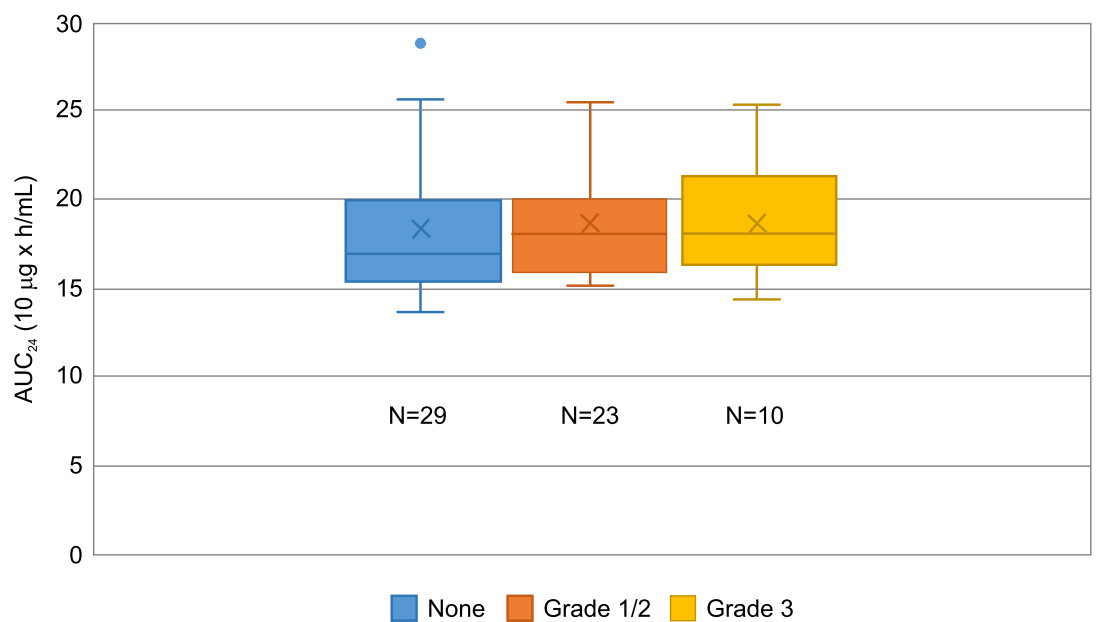

Fig. 4 Plasma-Exposure and Incidence of Rash: Pooled results of Japanese patients from SPARTAN and TITAN studies. a) Apalutamide, b) N-desmethyl apalutamide. Relationships between incidence of rash (by grade) and the plasma exposure ( $\mathrm{UUC}_{0-24, \text { ss }}$ ) to apalutamide or $\mathrm{N}$-desmethyl apalutamide were explored using boxplots

month following appropriate management by physicians. This could be attributed to the more frequent use of topical corticosteroids (77.1\%) and oral antihistamines (51.4\%) as supportive medication among Japanese patients when compared with patients in the global studies (combined data from SPARTAN and TITAN: topical corticosteroids, $37.8 \%$; antihistamines, $36.3 \%$.

Apalutamide and N-desmethyl apalutamide are two major pharmacologically active components detected in the systemic circulation at steady-state [8, 9]. The current integrated analysis showed that there was a possible correlation between plasma exposure to apalutamide and incidence of skin rash, but it did not significantly impact the grade of skin rash. However, the results should be interpreted with caution due to the limited sample size. The exposure-response modeling analysis for SPARTAN study based on whole study population was reported in a separate article [10].

Recently, the National Comprehensive Cancer Network $(\mathrm{NCCN})$ guidelines were updated to recommend apalutamide or enzalutamide, another second-generation antiandrogen, in patients with nmCRPC with background ADT therapy [11]. Skin rash was not observed to be an adverse drug reaction (ADR) in Phase 3 trials, either in nmCRPC or $\mathrm{MCRPC}$ patients $[12,13]$ with enzalutamide, although a case report of enzalutamide-induced acute generalized exanthematous pustulosis was recently reported and the USPI includes rash as an ADR observed in post-marketing [14]. Skin rash has also been reported with the firstgeneration oral non-steroidal antiandrogen drug 
bicalutamide and other new-generation oral antiandrogen drugs such as darolutamide [15-18]. It remains to be elucidated if skin rash is a class-effect, particularly in Japanese patients.

There is evidence to suggest that ethnic difference and genotypic heterogeneity between the Japanese and Caucasian populations could have an impact on treatment responses in patients with PC [19-21]. The correlation of human leukocyte antigen (HLA) typing with serious drug-induced skin rash e.g., StevensJohnson syndrome or toxic epidermal necrosis, is well established. However, further studies to explore the correlation of HLA typing with apalutamide-related skin rash are needed. Interestingly, Japanese men have been observed to respond better to hormonal therapy compared to their Caucasian counterparts [22, 23]. With reports of skin rashes being disproportionately higher in the Japanese subset of SPARTAN and TITAN studies and no clinical risk factors identified in the current study, further studies are needed to understand the basis of the high incidence observed.

To assess the clinical risk factors associated with commonly administered PC drugs, an analysis of AEs among nmCRPC patients treated with abiraterone (a synthetic, steroidal CYP17A1 inhibitor recently approved in the treatment of $\mathrm{PC}$ ), enzalutamide, or bicalutamide, was carried out in the real-world setting. Baseline AEs, Charlson comorbidity index, surgical castration, and older age were found to be significant predictors of AEs [24]. However, in our study, which was conducted based on registration trials, no clinical risk factors of skin rash were identified following apalutamide treatment. Therefore, it is important to analyze clinical risk factors of skin rash using real-world evidence.

A recent study assessed important safety factors that physicians considered in making treatment decisions for patients with nmCRPC. Among the safety attributes analyzed, which included incidence of skin rash, physicians were most concerned regarding cognitive problems, fractures, and fatigue. In fact, physicians regarded reduction in cognitive problems (from severe to none) to have a $36.0 \%$ higher importance in comparison to improving OS by 12 months instead of 3 months. Therefore, even though an important adverse event, skin rash was not a major determinant in physicians' choice of treatment for $\mathrm{PC}$, reiterating the need to assess the benefit-risk ratio to determine the best treatment for a particular patient with PC $[24,25]$.

Some of the baseline characteristics were not comparable between SPARTAN, TITAN and PCR1008 groups, with the median time from initial diagnosis, PSA levels, treatment durations, and ALP levels varying between these patient populations, since these were inherently different patient populations with different disease status at baseline. Although the cohorts were relatively dissimilar, the overall apalutamide regimens were similar amongst different disease populations in three studies. There may be an observer bias in an open-label Phase 1 study, however, it was included in the pooled analysis since the treatment dose $(240 \mathrm{mg} /$ day $)$ and the incidence of skin rash were similar to those in Phase 3 studies. In current research, we focussed on Japanese subpopulation for incidence of apalutamide-related skin rash. Most of skin rash occurred in the first 4 months after apalutamide initiation and it would be difficult to evaluate time to skin rash stratified by covariates since the patients' number is small. In order to conduct time-to-event analysis, larger apalutamide treatment data is warranted in the real-world settings.

The generalizability of the findings in this study is limited by the small number of patients analyzed $(N=68)$, its unplanned retrospective design, and the fact that the integrated analysis was done on different disease populations (nmCRPC, mCSPC and mCRPC). However, the results of this study underscore the need to undertake a prospective analysis of apalutamide-related skin rash in the Japanese population.

\section{Conclusions}

The incidence of apalutamide-related skin rash was higher in Japanese patients with PC compared to patients from the rest of the world. Although there is a potential correlation between incidence of skin rash and plasma exposure to apalutamide, it did not significantly impact the grade of skin rash. Moreover, no clinical risk factors associated with skin rash were identified. Most skin rash is observed within 120 days of treatment initiation and close monitoring of the skin rash, dose reductions/interruptions and treatment with oral antihistamines and topical and systemic corticosteroids led to resolution of the majority of skin rash observed in Japanese patients within 30 days.

\section{Supplementary information}

Supplementary information accompanies this paper at https://doi.org/10. 1186/s12894-020-00689-0.

Additional file 1. Supplementary Fig. 1. Kaplan-Meier plot for Time-to-Rash (All Grade).

Additional file 2. Supplementary Fig. 2. Kaplan-Meier plot for Time-to-Rash ( $\geq$ Grade 3).

Additional file 3. Supplementary Fig. 3. Kaplan-Meier plot for Time-toRemission of First Incidence of rash ( $\geq$ Grade 3 ).

Additional file 4. Supplementary Fig. 4. Kaplan-Meier plot for Time-toRemission of Maximum Grade Incidence of rash ( $\geq$ Grade 3).

Additional file 5. Supplementary Table 1. Incidence of Rash in SPAR TAN and TITAN.

Additional file 6. Supplementary Table 2. Time to Incidence of Rash, Rash Management, and Resolution of Rash in SPARTAN and TITAN. 


\section{Abbreviations}

ADR: Adverse drug reaction; ADT: Androgen-deprivation therapy; ALP: Alkaline phosphatase; AR: Androgen receptor; BSA: Body surface area; Cl: Confidence interval; ECOG PS: Eastern Cooperative Oncology Group Performance Status; HLA: Human leukocyte antigen; mCSPC: Metastatic castration-sensitive prostate cancer; mCRPC: Metastatic castration-resistant prostate cancer; MFS: Metastasis-free survival; NCCN: National Comprehensive Cancer Network; NCI CTCAE: National Cancer Institute Common Terminology Criteria for Adverse Events; nmCRPC: Non-metastatic castration-resistant prostate cancer; OS: Overall survival; PFS: Progression-free survival; PK: Pharmacokinetic; PSA: Prostate-specific antigen; QD: Once-daily; SD: Standard deviation

\section{Acknowledgements}

Anupama Singh, M. Tech, ISMPP, CMPPTM and Akshada Deshpande, PhD provided medical writing assistance and Sangita Patil, PhD, ISMPP, CMPP TM (all from SIRO Clinpharm Pvt. Ltd) provided additional editorial support for this manuscript.

\section{Authors' contributions}

HU1 was an investigator for SPARTAN, TITAN and PCR1008; HU2 was an investigator for SPARTAN and TITAN; both were involved in data collection for this study. YK and $Y$ I were involved in the design and analysis of this study. ALG and AS had a primary role in the study design and data interpretation for SPARTAN. KI and YI contributed to conception and design of PCR1008 study. All authors were involved in data interpretation, development, and critical revision of the manuscript for intellectual content. All authors met ICMJE criteria and all those who fulfilled those criteria are listed as authors. All authors had access to the study data and made the final decision about where to publish these data and approved submission to this journal.

\section{Authors' information}

Not applicable.

\section{Funding}

This analysis was conducted by Janssen Pharmaceutical K.K., Tokyo, Japan. The medical writing fee for this study was funded by Janssen Pharmaceutical K.K., Tokyo, Japan.

\section{Availability of data and materials}

The study materials and datasets used and/or analyzed during the current study are available from the corresponding author on reasonable request.

\section{Ethics approval and consent to participate}

Since this was a retrospective pooled analysis of 3 trials (SPARTAN, TITAN and PCR1008), Institutional Review Board approval to conduct this study was not required.

\section{Consent for publication}

\section{Not applicable.}

\section{Competing interests}

Dr. Hiroji Uemura received research grants from Janssen Pharmaceutical (during the conduct of the study) and lecture fees/subsidies from Bayer Yakuhin, Limited, Takeda Pharmaceutical Company Limited, AstraZeneca K.K., TAlHO Pharmaceutical Company Limited, Astellas Pharma Inc. and Pfizer Inc. Dr. Takeshi Kambara has no competing interests.

Dr. Hirotsugu Uemura received lecture fee from Pfizer Japan Inc., Ono pharmaceutical Co., Ltd., Bayer Yakuhin, Limited, Bristol-Myers Squibb, MSD K.K., research fee or grants from TAlHO Pharmaceutical Company Limited, Janssen Pharmaceutical K.K., Mebix, Inc., MSD K.K., Astellas Pharma Inc., Covence, Bayer Yakuhin, Limited, Prexel International Inc., Pfizer Japan Inc. ICON Japan K.K., Ono pharmaceutical Co., Ltd., Daiichi Ssankyo Company Limited, AstraZeneca K. K, EPS Corporation, Green Peptide, PPD-SNBL K.K., Takeda Pharmaceutical Company Limited, Chugai Pharmaceutical Co. Ltd., IQVIA, Mediscience Planning Inc., Osaka Urology Research Foundation, scholarship/ encouragement donations from Asahi Kasei Corporation, Kissei Pharmaceutical Co., Ltd., Novartis Pharmaceutical, Pfizer Japan Inc., Astellas Pharma Inc., Ono pharmaceutical Co., Ltd., Takeda Pharmaceutical Company Limited, Sanofi K.K., Daiichi Sankyo Co., Ltd.
The following authors are full-time employees of Janssen Pharmaceutical K.K. of Johnson \& Johnson: Yosuke Koroki, Yuki Iwaki, and Keiichiro Imanaka; and Janssen Research and Development: Andressa Smith and Angela LopezGitlitz.

\section{Author details}

'Department of Urology and Renal Transplantation, Yokohama City University Medical Center, Yokohama, Japan. ${ }^{2}$ Medical Affairs, Janssen Pharmaceutical K.K., Tokyo, Japan. ${ }^{3}$ Clinical Pharmacology, Janssen Pharmaceutical K.K, Tokyo, Japan. ${ }^{4}$ Clinical Science, Janssen Pharmaceutical K.K, Tokyo, Japan. ${ }^{5}$ Department of Dermatology, Yokohama City University Medical Center, Yokohama, Japan. ${ }^{6}$ Janssen Global Research \& Development, Spring House, PA, USA. ${ }^{7}$ Department of Urology, Kindai University Faculty of Medicine, Osaka, Japan.

Received: 5 May 2020 Accepted: 29 July 2020

Published online: 02 September 2020

\section{References}

1. Clegg NJ, Wongvipat J, Joseph JD, et al. ARN-509: a novel antiandrogen for prostate cancer treatment. Cancer Res. 2012;72:1494-503.

2. Rice MA, Malhotra SV, Stoyanova T. Second-generation antiandrogens: from discovery to standard of Care in Castration Resistant Prostate Cancer. Front Oncol. 2019;9:801.

3. Smith MR, Saad F, Chowdhury S, et al. Apalutamide treatment and metastasis-free survival in prostate cancer. N Engl J Med. 2018:378:1408-18.

4. Chi KN, Agarwal N, Bjartell A, et al. Apalutamide for metastatic, castrationsensitive prostate cancer. N Engl J Med. 2019;381:13-24.

5. Uemura $H$, Satoh $T$, Tsumura $H$ et al. Efficacy and safety of apalutamide in Japanese patients with non-metastatic castration-resistant prostate cancer: A subgroup analysis of a randomized, double-blind, placebo-controlled, phase 3 study. Prostate Int. 2020. https://doi.org/10.1016/j.prnil.2020.05.002.

6. Tsuchiya $T$, Imanaka K, Iwaki $Y$, et al. An open-label, phase 1 study of androgen receptor antagonist, apalutamide in Japanese patients with metastatic castration-resistant prostate cancer. Int J Clin Oncol. 2019;24: 1596-604.

7. Pérez-Ruixo C, Pérez-Blanco JS, Chien C, et al. Population pharmacokinetics of apalutamide and its active metabolite $\mathrm{N}$-desmethyl-apalutamide in healthy and castration-resistant prostate cancer subjects. Clin Pharmacokinet. 2020;59:229-44

8. Rathkopf DE, Morris MJ, Fox JJ, et al. Phase I study of ARN-509, a novel antiandrogen, in the treatment of castration-resistant prostate cancer. J Clin Oncol. 2013;31:3525-30.

9. Alkhudair NA. Apalutamide: emerging therapy for non-metastatic castrationresistant prostate cancer. Saudi Pharm J. 2019;27:368-72.

10. Perez-Ruixo C, Ackaert O, Ouellet D et al. Efficacy and safety exposureresponse relationships of apalutamide in patients with non-metastatic castration-resistant prostate cancer. Clin Cancer Res. 2020. https://doi.org/ 10.1158/1078-0432.CCR-20-10412020.

11. NCCN. Prostate Cancer. https://www.nccn.org/patients/guidelines/content/ PDF/prostate-patient.pdf. Accessed on 10 Oct 2019.

12. Hussain M, Fizazi K, Saad F, et al. Enzalutamide in men with nonmetastatic, castration-resistant prostate cancer. N Engl J Med. 2018;378:2465-74.

13. Beer TM, Armstrong AJ, Rathkopf DE, et al. Enzalutamide in metastatic prostate cancer before chemotherapy. N Engl J Med. 2014;371:424-33.

14. Alberto C, Konstantinou MP, Martinage C, et al. Enzalutamide induced acute generalized exanthematous pustulosis. J Dermatol Case Rep. 2016;10:35-8.

15. Lee S, Chung YJ, Kim BH, et al. Comparative pharmacokinetic evaluation of two formulations of bicalutamide 50-mg tablets: an open-label, randomized-sequence, single-dose, two-period crossover study in healthy Korean male volunteers. Clin Ther. 2009;31:3000-8.

16. Chodak G, Sharifi R, Kasimis B, et al. Single-agent therapy with bicalutamide: a comparison with medical or surgical castration in the treatment of advanced prostate carcinoma. Urology. 1995;46:849-55.

17. Kolvenbag GJCM, Blackledge GRP. Worldwide activity and safety of bicalutamide: a summary review. Urology. 1996;47:70-9.

18. Fizazi K, Shore N, Tammela TL, et al. Darolutamide in nonmetastatic, castration-resistant prostate cancer. N Engl J Med. 2019;380:1235-46.

19. Irvine RA, Yu MC, Ross RK, Coetzee GA. The CAG and GGC microsatellites of the androgen receptor gene are in linkage disequilibrium in men with prostate cancer. Cancer Res. 1995;55:1937-40. 
20. Suzuki H, Akakura K, Komiya A, et al. CAG polymorphic repeat lengths in androgen receptor gene among Japanese prostate cancer patients: potential predictor of prognosis after endocrine therapy. Prostate. 2002;51: 219-24.

21. Sartor $\mathrm{O}$, Zheng $\mathrm{Q}$, Eastham JA. Androgen receptor gene CAG repeat length varies in a race-specific fashion in men without prostate cancer. Urology. 1999:53:378-80

22. Fukagai T, Namiki TS, Carlile RG, et al. Comparison of the clinical outcome after hormonal therapy for prostate cancer between Japanese and Caucasian men. BJU Int. 2006;97:1190-3.

23. Namiki M, Ueno S, Kitagawa Y. Role of hormonal therapy for prostate cancer: perspective from Japanese experiences. Transl Androl Urol. 2012;1:160-72.

24. Shah A, Hussain A, Shah R, et al. Predictors and rates of adverse events (AEs) among non-metastatic prostate cancer (nmPC) patients (pts) treated with bicalutamide, abiraterone, or enzalutamide following surgical/medical castration. J Clin Oncol. 2019:37:e16556.

25. Mateo J, Fizazi K, Gillessen S, et al. Managing nonmetastatic castrationresistant prostate cancer. Eur Urol. 2019;75:285-93.

\section{Publisher's Note}

Springer Nature remains neutral with regard to jurisdictional claims in published maps and institutional affiliations.

Ready to submit your research? Choose BMC and benefit from:

- fast, convenient online submission

- thorough peer review by experienced researchers in your field

- rapid publication on acceptance

- support for research data, including large and complex data types

- gold Open Access which fosters wider collaboration and increased citations

- maximum visibility for your research: over $100 \mathrm{M}$ website views per year

At $\mathrm{BMC}$, research is always in progress.

Learn more biomedcentral.com/submissions 